\section{Rupture of the Tendo Achillis}

A tea-planter, aged 38, was playing deck tennis on the homeward voyage when he felt a pain in his left leg, and found that he was quite unable to stand on tip-toe. He was so pleased to be home again that his condition was not diagnosed until four months after the accident, and valuable time had been lost.

At operation, on May 20th, 1931, it was found that a gap of $1 \frac{1}{2}$ inches in the tendo Achillis had been bridged by fibrous tissue. This was excised and the calf muscles mobilized, without, however, making direct apposition possible. The knee and anklo were flexed, but there was still a small gap to bridge. A length of $3 \frac{1}{2}$ inches of half the peroneus longus tendon was passed through the two ends of the tendo Achillis, and a part of the proximal end of the tendo Achillis was turned down and sutured to its distal part. No attempt was made to close the sheath, and there was considerable difficulty even to get the skin together. The limb was put in plasterof-Paris with the knee fully flexed and the ankle plantarflexed. The knee was extended in a week, and the foot brought to 110 degrees two weeks later, when the stitches were removed and the plaster changed.

Eight weeks from the operation the patient was walking in an ordinary shoe, with the heel raised half an inch, but no plaster or apparatus. When he was seen four months after the operation he was wearing an ordinary heel, his foot was at 90 degrees, 'and he could stand on tip-toe on the affected side, bearing his whole weight on that foot for a fraction of a second. He had experienced no trouble, except for a stitch abscess, which was no doubt due to the mistake of using silk instead of catgut.

\section{Bilateral Rupture of the Quadriceps Tendon}

On March 18th, 1932, a Jewish shopkeeper, aged 77, and weighing 16 st., caught his heel on the stairs and slipped.

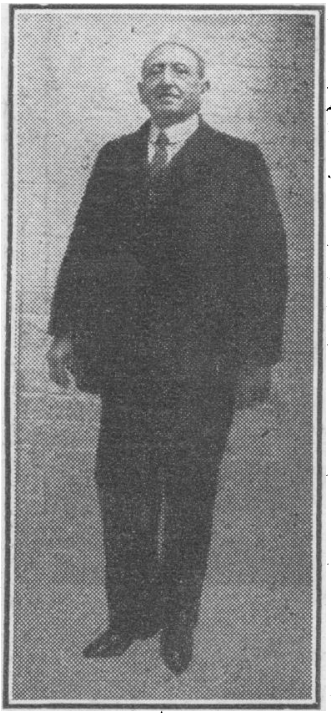

He had been unable to extend his knees since the accident, and walking was out of the question. He was very depressed by reason of a gloomy prognosis, based on the assumption that he had a haematomyelia of the cord and would never walk again.

At operation on the right side, on June 6th, 1932 (two and a half months after the accident), the quadriceps tendon was found to be torn at the actual point of insertion into the patella, making it necessary to drill a hole in the patella and suture the tendon with strong silk. This suture line was reinforced with fascia lata, and the capsule of the joint closed with catgut. Two weeks later the left side was operated upon, and a complete rupture disclosed and repaired.

A year later the patient was Fig. 3. - Case mir. seen at his little shop in Holborn ; Bilateral rupture of quadri- he could walk without sticks, and ceps tendon. Sixteen-stone had active extension of the knee patient, aged 79, nearly through 30 degrees. He did, howand reinforced suture. ever, generally use sticks for Operation two and a half walking, as he felt somewhat months after accident. insecure, no doubt partly owing to He can stand unaided, as his age and weight.
shown.

\section{Comment on Delayed and Reinforced Tendon Suture}

The successful results in these three cases are no argument for delay in suturing cut or ruptured tendons. The diagnosis of such an injury ought to be followed immediately by operation in any recent case. If, however, diagnosis or treatment has been delayed, there is still a good chance of getting a satisfactory result, even two or three months after an injury of the large extensor tendons. The story is an altogether different one for the smaller tendons, which are concerned with fine movement, such as those of the hand.

\section{Operative Technique}

The exact operation will vary in different cases, but the following points in technique seem reasonable.

1. The functional efficiency of a muscle demannds apposition of the torn ends of the tendon whenever possible and, failing that, the shorter the bridge the stronger will be the muscle.

2. Aids in securing apposition. Posture of adjacent joints and mobilization of muscle bellies help, but good honest pulling on the muscle generally helps more.

3. Suture material. Strong chromic catgut is the best, but it must be supported by splint or plaster-of-Paris for six weelss. Weight-bearing may be allowed during the latter half of the period if the sutured tendon is in the lower limb.

4. Supplementary sutures. Peroneal tendon and fascia lata will sometitnes turn doubt into security. They are always available in case of need.

5. The immediate post-operative position of a joint should relax the suture line, but if such is a malposition it must be gradually corrected in two or three weeks if the patient is an adult. In children this is not so essential.

6. It is much better to put the tendon through a hole in the bone than to stitch it to periosteum.

\section{A SECOND ATTACK OF ACUTE POLIOMYELITIS}

\author{
BY
}

THOMAS MOORE, M.B., B.S.

CLINICAL ASSISTANT TO THE ORTHOPAEDIC DEPARTMENT, ROYAL VICTORIA INFIRMIARY, NEWCASTLE-UPON-TYNE

The occurrence of a second attack of acute anterior poliomyelitis is rare.* In 1930 Still $^{1}$ reported one case, and was only able to collect eight definite cases from the literature. The diagnosis depends entirely on clinical evidence. In the case here reported this seems to be so complete as to leave no doubt as to the diagnosis.

\section{Case Record}

History.-On February 14th, 1934, W. L., aged 7 years was brought to the out-patient department of the-Royal Victoria Infirmary, Newcastle, because he was paralysed. His mother volunteered the following history. The child was healthy at birth; but at 10 months of age he had an attack of pneumonia. He was ill for fourteen days. After this the lower limbs seemed to " pine away," and he did not progress normally with learning to walk, as he had been doing previously. When the child was 3 years old he walked so badly - " his feet just seemed to flop about." - that his mother took him to see Dr. Nattrass at the Royal Victoria Infirmary (April 5th, 1930). He was given massage treatment and fitted with iron splints. He was able to walk remarkably well in these, used to go to school in them, and lived as a normal boy until eight weeks ago. He was then sent home from school because he was not well. The mother noticed that he wás " heavy" and feverish, and his nose was running. A doctor who was called in thought he was sickening for measles. In a day or two, however, he improved, and returned to school after a week's absence. A week later the child was taken ill again, and had to be put to bed. On the third day a rash appeared, which was diagnosed as measles. The child became very weak and helpless, and unable to feed himself. He complained of pains in his back and limbs, which were sore to the touch. After fourteen days an attempt was made to get the child up. It was then found he could neither stand nor sit.

* A short case record appeared in the Epitome of Current Medical Literature of June 9th, 1934, para. 474. 
Clinical Findings (February 14th, 1934).-Examination revealed a rather pale child of normal intelligence. No abnormality could be detected on examination of the respiratory, cardiovascular, or alimentary systems. The cranial nerves were found to be normal. Examination of the upper limbs was as follows. The proximal muscles were normal. In both hands there was marked wasting of the interossei, and of the thenar and hypothenar eminences, so that the hands appeared flattened. The fingers were held in slight flexion at the interphalangeal joints, and hyperextension at the metacarpophalangeal joints. The power of flexion of the wrists and fingers was weak. There was no power at all in the left flexor carpi ulnaris. The patient was unable to extend the interphalangeal joints of the fingers or flex the metacarpophalangeal joints, and attempts to do so only resulted in an increase of the claw-hand deformity. He was able to extend and flex the metacarpo-phalangeal and the interphalangeal joints of the thumbs. There was no power of adduction or of opposition of the thumbs. There was marked wasting of both lower limbs, and each showed the deformity of talipes equinovarus. There was some cutaneous hyperaesthesia to touch of the left lower limb. All the muscles moving the hip-joints were very much reduced in power. Both quadriceps and hamstring muscle groups were very weak, particularly on the left side. All the muscles moving ankle and midtarsal joints were completely paralysed save the left posterior tibialis, which had a trace of power. There was no power in the recti abdominis.

Electrical Reactions (April 24th, 1934).-There was no faradic response in the muscles of the legs and (?) no galvanic response. There was no faradic response in flexors of the right wrist or fingers, but some in the flexor carpi radialis. No faradic response occurred in the muscles of the right hand. There was fair galvanic response. Faradic response was present in all flexors of the left wrist and fingers, there being no faradic response in the muscles of the left hand. The galvanic response was fair.

It was particularly fortunate that this child was originally seen in the hospital, for the following clinical findings, made on April 5th, 1930, are available. "A case of anterior poliomyelitis-atrophic weakness of both lower limbs, double paralytic talipes equinovarus." The electrical reactions recorded at the same time are given as follows. "Right leg-no faradic response in extensor digitorum longus, extensor hallucis longus, or peronei ; good response in anterior and posterior tibiales; faint response in calf muscles; galvanic response rather poor. Left leg-no faradic response in anterior tibialis or peroneal muscles ; good in calf muscles ; long flexor doubtful ; galvanic response rather poor."

The child was given the usual physical treatment, and was fitted with double irons to the right knee and full-length irons on the left leg attached to pelvic band.

\section{Conclusion}

From these findings there appears to be no doubt that the child, at an early age, had an attack of acute anterior poliomyelitis. This left him with some weakness of the lower limbs, but he was able to walk remarkably well in supports, and lived as a normal boy until nearly 7 years of age. Then, following two rather indefinite febrile illnesses, the muscular weakness became much more widespread. The affection of the lower limbs increased in severity, and the hands and recti abdominis were affected in addition. In consequence the child was left in a very helpless condition. While he has been in hospital the condition of the limbs has not improved, but the recti abdominis are recovering slowly. There would appear to be no other explanation of these findings but that a second attack of acute anterior poliomyelitis has occurred.

My thanks are due to Mr. Gordon Irwin, under whose care the patient is, for permission to publish the case, and to Dr. F. J. Nattrass for his helpful advice and criticism.

\section{Reference}

1 Still, G. F.: Second Attacks of Acute Poliomyelitis and the Minimal Duration of Immunity, Arch. Dis. Child., 1930, v, 291.

\section{Clinical Memoranda}

\section{A MODIFICATION OF BENEDICT'S TEST}

In most laboratories either Fehling's reagent ${ }^{1}$ or Benedict's qualitative reagent ${ }^{1}{ }^{2}$ is used in making a preliminary rough estimate of the amount of sugar in the urine, but with the application described below I have been able to dispense with the standard quantitative determinations altogether in routine clinical work. The method depends on observing the colour of the filtrate obtained after boiling the urine with Benedict's qualitative reagent. By experiment it was found that $5 \mathrm{c.cm}$. of the reagent is just decolorized by $0.5 \mathrm{c} . \mathrm{cm}$. of 2 per cent. glucose (that is, $10 \mathrm{mg}$.) in five minutes in a boiling-water bath.

\section{TEChNique OF TEST}

Five c.cm. of Benedict's qualitative reagent and $0.5 \mathrm{c.cm}$. of urine are mixed in a test tube, which is placed in a boilingwater bath for exactly five minutes. The mixture is filtered hot through a fine paper (Whatman No. 44) into a tube of the same bore as that of the standard colour tubes. The colour of the unknown is then compared with that of the standards, the tubes being observed against a white background. If the filtrate is colourless 2 per cent. or more of glucose is present: the test is therefore repeated, employing urine which has been diluted twofold or fourfold, and allowing for the dilution in the calculation.

The preparation of the standard colour tubes and the concentration of sugar to which each corresponds will be clear from the following table.

\begin{tabular}{c|c|c|c}
\hline $\begin{array}{c}\text { No of } \\
\text { Standard } \\
\text { Tube }\end{array}$ & $\begin{array}{c}\text { Amount of } \\
\text { Benedict's Solution } \\
\text { (c.cm.) }\end{array}$ & $\begin{array}{c}\text { Amount of } \\
\text { Distilled Water }\end{array}$ & $\begin{array}{c}\text { Percentage of } \\
\text { Glucose to which } \\
\text { Tube Corresponds }\end{array}$ \\
\hline 1 & 5 & 0.5 & 0.0 \\
2 & 4 & 1.5 & 0.4 \\
3 & 3 & 3.5 & 0.8 \\
4 & 2 & 4.5 & 1.2 \\
5 & 1 & 5.5 & 1.6 \\
6 & 0 & & 2.0 or over \\
\hline
\end{tabular}

The above data have been established by direct experiment. Thus it was found that the colour of the filtrate, after heating 5 c.cm. of reagent and $0.5 \mathrm{c} . \mathrm{cm}$. of 0.4 per cent. glucose in a boiling-water bath for five minutes, exactly matched the colour of a mixture of $4 \mathrm{c.cm}$. of reagent and $1.5 \mathrm{c} . \mathrm{cm}$. of water; and so on for the other tubes. The filtrate may have a greenish tinge which, when marked, renders matching with the blue standards rather difficult. Nevertheless, the accuracy that can be attained by this modification is always sufficient for clinical purposes. The only special apparatus required is a set of six uniform tubes containing the standard solutions. These can be readily prepared from the table given above.

I wish to thank Dr. G. A. Harrison for providing the facilities for doing this work in the Laboratory of Chemical Pathology at St. Bartholomew's Hospital.

A compact outfit, comprising a set of standards fixed in a suitable stand, filter paper (No. 44), small filter funnels, and tubes uniform with those containing the standard solutions, can be obtained from British Drug Houses, Ltd., Graham Street, London, N.1.

St. Bartholomew's Hospital. Joseph Fine, M.D.

\section{REFERFNCES}

'Harrison: Chemical Methods in Clinical Medicine, 1930, i, 96 ; ii, 85.

'Beaumont and Dodds: Recent Advances in Medicine, 1934, p. 103. 